\title{
Cost-Effectiveness Analysis of Qsymia for Weight Loss
}

\author{
Eric A. Finkelstein · Eliza Kruger • \\ Sunil Karnawat
}

Published online: 2 July 2014

(c) The Author(s) 2014. This article is published with open access at Springerlink.com

\begin{abstract}
Background Phase 3 clinical trial results reveal that Qsymia is a clinically effective long-term treatment for obesity, but whether this treatment is cost-effective compared to a diet and lifestyle intervention has yet to be explored.

Objective To quantify the incremental cost-effectiveness of Qsymia (phentermine and topiramate extended-release) for health-related quality of life improvements.

Study design and methods Estimates are based on cost and quality of life outcomes from a 56-week, multicenter, placebo-controlled, phase 3 clinical trial undertaken in 93 health centers in the US. Participants were overweight and obese adults (aged 18-70 years) with a body-mass index of $27-45 \mathrm{~kg} / \mathrm{m}^{2}$ and two or more comorbidities (hypertension, dyslipidemia, diabetes or pre-diabetes or abdominal obesity). The intervention was diet and lifestyle advice plus the recommended dose of Qsymia (phentermine $7.5 \mathrm{mg}$ plus topiramate $46.0 \mathrm{mg}$ ) vs. control, which included diet and lifestyle advice plus placebo. The study was from the payer perspective. Costs included the prescription cost, medication cost offsets and physician appointment costs.
\end{abstract}

Electronic supplementary material The online version of this article (doi:10.1007/s40273-014-0182-6) contains supplementary material, which is available to authorized users.

E. A. Finkelstein $(\square) \cdot$ E. Kruger

Duke-NUS Graduate Medical School, 8 College Road,

Singapore, Singapore

e-mail: eric.finkelstein@duke-nus.edu.sg

E. A. Finkelstein

Duke University Global Health Institute, Durham, NC, USA

S. Karnawat

VIVUS, Inc., Mountain View, CA, USA
Effectiveness was measured in terms of quality-adjusted life years gained (QALYs). The main outcome measure was incremental cost per QALY gained of the intervention relative to control.

Results Our base-case model, in which participants take Qsymia for 1 year with benefits linearly decaying over the subsequent 2 years, generates an incremental cost-effectiveness ratio (ICER) of $\$ 48,340$ per QALY gained. Using the base-case assumptions, probabilistic sensitivity analyses reveal that the ICER is below $\$ 50,000$ per QALY in $54 \%$ of simulations. However, results are highly dependent on the extent to which benefits are maintained post medication cessation. If benefits persist for only 1 year post cessation, the ICER increases to $\$ 74,480$.

Conclusion Although base-case results suggest that Qsymia is cost-effective, this result hinges on the time on Qsymia and the extent to which benefits are maintained post medication cessation. This should be an area of future research.

\section{Key Points for Decision Makers}

Given the obesity epidemic, decision makers are looking to identify cost-effective strategies to improve the health of their obese members.

Few effective medical interventions for weight loss are available.

These results show that, in addition to clinically significant weight loss, Qsymia generates improvements in quality of life and reductions in medication costs.

If benefits persist beyond medication cessation, results suggest that Qsymia may be a cost-effective nonsurgical alternative for weight management. 


\section{Introduction}

Obesity is a major risk factor for a number of adverse health conditions, including type 2 diabetes, heart disease, and cancer [1]. Further, worldwide obesity rates have more than doubled in the past 3 decades, such that excess weight is now the fifth leading risk for global deaths, accounting for roughly 2.8 million deaths each year [2].

In addition to the health effects, obesity imposes significant external costs on society. For example, in the United States (US) alone, annual medical expenditures for treating obesity-related health conditions now exceed $\$ 147$ billion per year, with roughly half of this total directly financed by Medicare and Medicaid [3]. Other research shows that an obese worker has annual direct and indirect costs that are between $\$ 1,143$ (class I obese male) and $\$ 6,694$ (class II obese female) greater than costs for their normal-weight counterparts, depending on gender and degree of excess weight [4]. Moreover, the indirect costs of obesity, which result from workloss due to absenteeism and presenteeism, exceed the direct medical costs.

Due to these impacts of obesity on society, there are significant health and economic benefits that could be accrued to individuals, governments, insurers, and employers through successful weight-loss initiatives. Typically, the first line of treatment for excess weight is lifestyle modification [5]. However, on average, lifestyle interventions generate only modest weight loss [6], and this weight loss is rarely maintained long term [7]. Bariatric (weight loss) surgery is an effective treatment for severe obesity [8], but due to its high cost (at least $\$ 20 \mathrm{~K}$ for the surgery alone) and invasive nature, few obese individuals have opted for this strategy. Roughly 113,000 weight-loss surgeries are performed yearly in the US [9], and yet this number is dwarfed by the obese population-estimated to be over 80 million adults $[10,11]$.

Until recently, pharmacologic interventions for addressing chronic weight management have been limited. The drug combination fenfluramine/phentermine, referred to as fen-phen, was the first commercially successful weight loss drug. Although it was highly effective, it was later shown to have potentially fatal side effects, which led to its withdrawal from the market in 1997 [12]. Over the next 15 years, only two weight-loss prescription medications were widely available on the US market, sibutramine (Meridia) and orlistat (Xenical). Sibutramine was subsequently pulled from the market due to increased risk of cardiovascular events [13]. Until 2012, only orlistat was available for chronic use, but the weight loss observed in clinical trials was only a few percentage points greater than that seen with a placebo, and is associated with gastrointestinal side effects that limit its appeal $[14,15]$.
However, in 2012, the US FDA approved two new therapies for chronic weight management: Belviq (lorcaserin) and Qsymia (phentermine plus topiramate extendedrelease), to be used in combination with diet and exercise. In a 1-year clinical trial, Qsymia phentermine 7.5 and topiramate $46 \mathrm{mg}$ (recommended dose per label) [16], in conjunction with diet and exercise, generated a clinically significant weight loss of $6.6 \%$ of body weight relative to diet and exercise plus placebo [17]. Statistically significant improvements with the Qsymia recommended dose compared with placebo were also noted in blood pressure, waist circumference, lipids, glycemic parameters, and inflammatory biomarkers. Moreover, rates of discontinuation with Qsymia were lower than with placebo, suggesting Qsymia has a tolerable side-effect profile. A follow-up study showed that weight loss was maintained after 2 years [18].

These results suggest that Qsymia is effective at inducing weight loss and improving risk factors associated with comorbidities. However, the incremental cost-effectiveness of Qsymia compared to diet and exercise alone remains unknown. Therefore, the objective of this study was to assess the long-term cost-effectiveness of the recommended dose of Qsymia from the payer perspective. Although the Centers for Medicare and Medicaid Services (CMS) has maintained a policy of not considering costeffectiveness in national coverage decisions $[19,20]$, private insurers and large employers do not face such a restriction and have relied on cost-effectiveness analyses to inform decision making. For example, UnitedHealth Group now provides coverage for health coaches at YMCAs to work with overweight patients at high risk for type 2 diabetes based on research showing that this approach is likely to be cost-effective [21]. Because of pressure to provide pharmacologic treatments for obesity as a covered benefit, some insurers/employers will be interested to know whether long-term treatment on Qsymia is likely to be costeffective.

\section{Methods}

\subsection{Study Description}

This study utilized the effectiveness results from the CONQUER randomized controlled trial. A complete description of the trial is available elsewhere [17]. In brief, CONQUER was a 56-week randomized, double-blind, placebo-controlled trial conducted at 93 health centers in the USA to determine the effectiveness of Qsymia for weight loss and weight-related comorbidities. The trial was undertaken between November 1, 2007 and June 30, 2009. Adult participants were eligible if they were overweight or 
obese with a body-mass index (BMI) between 27 and $45 \mathrm{~kg} / \mathrm{m}^{2}$ and at least two obesity related co-morbidities, including increased waist circumference, elevated blood pressure, elevated cholesterol or type 2 diabetes managed with lifestyle modification or metformin. For those with type 2 diabetes, no lower BMI limit was applicable. Full inclusion and exclusion criteria were previously published [17].

Eligible participants were stratified by gender and diabetes status and then randomly assigned in a 2:1:2 ratio to the placebo arm, the phentermine $7.5 \mathrm{mg}$ plus topiramate extended-release $46 \mathrm{mg}$ arm (Qsymia recommended dose), and the phentermine $15 \mathrm{mg}$ plus topiramate extendedrelease $92 \mathrm{mg}$ arm (Qsymia top dose) for 56 weeks. All participants received recommended counseling for diet and lifestyle modification. Study visits occurred at baseline and weeks 2 and 4 during drug titration, and every 4 weeks thereafter.

\subsection{Effectiveness}

Prior analyses [17] focused on quantifying the efficacy and safety of Qsymia doses. Co-primary end-points were mean weight loss from baseline and the percentage who achieved clinically significant weight loss, defined as $5 \%$ or greater from baseline. We extended that analysis by computing the incremental cost-effectiveness ratio of the recommended dose of Qsymia relative to placebo in terms of cost per quality-adjusted life year (QALY) gained. We focused on the incremental cost-effectiveness of the recommended dose as it is the most commonly prescribed dose of Qsymia [22].

This analysis was conducted from the payer perspective, which included the direct cost of Qsymia (assuming no rebates, co-payments or deductibles) less cost offsets from reductions in concomitant medications. The primary costeffectiveness analysis was based on the intention-to-treat (ITT) sample, which consisted of all participants who completed baseline measurements and surveys, were randomly assigned, took at least one dose of the study drug or placebo, and had one post-baseline bodyweight measurement. Completers included a subset of these individuals who completed the trial on the study drug or placebo and for whom the final end-point measurement was obtained within 7 days of the last dose (see the Electronic supplementary material, ESM).

The primary measure of effectiveness was an imputed health-related quality of life (QoL) score which was derived from the Short Form-12 version 2 (SF-12v2) survey [23]. The SF-12v2 survey is a validated questionnaire used to assess participant physical and mental health. We calculated changes in the physical component summary (PCS) and mental component summary (MCS) scales based on the SF-12v2 survey. We used the Brazier and Roberts algorithm [24] to convert the SF-12v2 survey into a health-related QoL metric for each participant at baseline and at 7 and 12 months (where $0=$ dead and $1.0=$ best imaginable health state). This algorithm is widely used in cost-effectiveness analyses [25-28]. We then estimated the change in this metric over the trial duration, with the last observation carried forward (LOCF) for those with missing data. We employed a linear regression model with change in QoL as the dependent variable and treatment arm as the key outcome variable; sex and mean-centered baseline QoL were included as potential confounders. Analogous regressions were run for physical and mental health summary scores. All regression analyses were conducted using Stata 13.1 [29].

As it is not known when the QoL benefits accrued during the trial, we assumed that $50 \%$ of the full benefits accrue in the first year (i.e., they received 6 months of the benefit). We further assumed that the benefits linearly decayed after the medication was ceased. Our base case assumed that individuals were on Qsymia for 1 year, with benefits linearly decaying to zero 2 years post-drug cessation. In one-way sensitivity analyses, we assumed linear decays of 0,1 , and 3 years. We also modeled an additional scenario whereby patients remain on Qsymia for 2 years, with $50 \%$ of the benefits in the first year and full benefits in the second year, which then linearly decline to zero in years three and four. The assumption of full benefits through year two is supported by a follow-up study of Qsymia which found that individuals who remained on Qsymia for 2 years maintained the weight loss [18].

\subsection{Costs}

Costs and potential cost offsets for this analysis were limited to direct costs of Qsymia, physician appointments, and any potential cost offsets from reducing medications for concomitant medications. Based on market prices (September 2013), the direct cost of the recommended dose of Qsymia is \$5.12 per day [30]. Noting that a large percentage stopped taking their medication mid-trial $(31 \%$ in the Qsymia recommended dose compared to $43 \%$ in placebo), costs were allocated to participants according to how long each remained on the medication during the first 52 weeks of the 56-week trial period. We then included costs for two physician visits that would be expected to occur in the first year that someone starts a prescription weight loss drug [31].

\subsection{Medication Cost Offsets}

We focused on cost offsets for medications used to treat dyslipidemia, hypertension, and type 2 diabetes, as these 
medications are likely to be influenced by successful weight loss and were tracked as part of the CONQUER trial [17]. Participants reported daily prescription medication use (dosage and name of drug) at baseline and study conclusion. Using this information, daily medication costs were quantified by multiplying the usage data by unit costs for each of the reported medications. Unit costs were obtained from Medi-Span's PriceRx database in January 2013. The wholesale acquisition cost (WAC) was used for branded medications, while the average WAC (AWAC) was used for unbranded generics. Participants with no utilization at baseline and 56 weeks were allocated a cost of zero dollars. Baseline carried forward was used for those missing costs at 56 weeks. We then computed the change in daily medication costs for each class of drug and for the three combined from baseline to 56 weeks. To identify the impact of Qsymia on daily medication costs, we ran the same regression as for QoL, using an ITT approach. Similar to QoL, we did not know the exact date that changes in medication occurred; therefore, we assumed that daily medication savings began at month six. In future years we assumed that all savings in concomitant medications accrue for the entire year if participants remained on Qsymia.

\subsection{Incremental Cost-Effectiveness (ICER) Ratios}

We calculated incremental cost effectiveness ratios by dividing the incremental costs of the intervention by the incremental benefits (QALYs). As noted above, our base case assumed individuals remained on the medication for 1 year and accrued benefits through year three. We also considered 2 years on Qsymia, with residual benefits accruing from years three through four. We applied a discount rate of $3.5 \%$ for all out-year estimates.

\subsection{Sensitivity Analysis}

Several sensitivity analyses were carried out. In one-way sensitivity analyses, key inputs were varied one at a time to simulate the impact on the incremental cost-effectiveness ratio. Specifically, we assessed the impact of:

1. Changing the duration of benefit decay post drug cessation from 2 years in the base case to either 0 (worst case), 1, or 3 years (best case),

2. Two years on Qsymia, with $50 \%$ of benefits in the first year and full benefits in the second year, linearly declining to zero in years three and four,

3. Using the lower and upper $95 \%$ confidence intervals of effectiveness as estimated using converted SF-12v2 data from the trial (results available in Table 1 of the ESM),

4. Varying prescription costs for Qsymia by $\pm 25 \%$,
5. Excluding the medication offset,

6. Changing the discount rate from 3.5 to $1 \%$ and $5 \%$, and

7. Assuming no residual benefits (or costs) beyond the trial period.

Subsequently, we employed probabilistic sensitivity analyses on the base-case model and ran the model 1,000 times to assess the effect of uncertainty regarding the input parameters (input values and a description of the calculations performed are available in the ESM). We assumed a normal distribution for medication costs and for effectiveness, using the estimated mean and standard deviations from the trial data. Based on the results, we report the percentage of iterations that fall below the $\$ 50,000$ per QALY threshold and the cost per QALY value which $95 \%$ of the observations fall below.

\section{Results}

\subsection{Study Sample}

Participant demographics are presented in Table 1. At baseline, the majority of participants were female and the average age was 51 years. Concerning comorbidities, $53 \%$ of participants had hypertension, $36 \%$ had dyslipidemia, and $16 \%$ of the placebo group and $13.7 \%$ of the Qsymia group had type 2 diabetes. At baseline, average weight was roughly $103 \mathrm{~kg}$ and average BMI was $36.5 \mathrm{~kg} / \mathrm{m}^{2}$. Daily medication expenses were on average $\$ 1.19$ and $\$ 1.37$ for hypertension, $\$ 1.21$ and $\$ 1.38$ for dyslipidemia, and $\$ 0.08$ and $\$ 0.07$ for type 2 diabetes for the placebo and Qsymia arms, respectively. In total, this amounted to an average $\$ 2.47$ and $\$ 2.83$ a day in medication expenses for these conditions for the placebo and Qsymia arms, respectively, at baseline. There were no significant differences in demographics or medication costs between the study arms at baseline.

\subsection{Effectiveness}

As presented previously, patients receiving the Qsymia recommended dose lost significantly more weight over the intervention period than those receiving placebo [17]. On average, participants taking the Qsymia recommended dose had $6.7 \mathrm{~kg}$ greater weight loss than those on the placebo $(p<0.01)$ [17]. At baseline, there was no difference in average PCS and MCS between groups (Table 2). Over the 56 weeks, both groups saw improvements in PCS scores, yet the adjusted change in PCS was 2.28 points higher for the Qsymia recommended group compared to the placebo group $(p<0.01$; Table 2). There were no significant treatment effects observed for MCS for either group. At baseline, using 
Table 1 Baseline characteristics of ITT sample, mean (SE)

\begin{tabular}{lcc}
\hline & $\begin{array}{l}\text { Placebo } \\
(n=979)\end{array}$ & $\begin{array}{l}\text { Qsymia recommended } \\
\text { dose }(n=488)\end{array}$ \\
\hline Age (years) & $51.2(0.33)$ & $51.1(0.47)$ \\
Women $(\%)$ & $70.3(0.01)$ & $69.9(0.02)$ \\
Baseline weight $(\mathrm{kg})$ & $103.3(0.58)$ & $102.8(0.82)$ \\
Body mass index $\left(\mathrm{kg} / \mathrm{m}^{2}\right)$ & $36.7(0.15)$ & $36.3(0.2)$ \\
Co-morbidities & & \\
Hypertension $(\%)$ & $52.7(0.02)$ & $52.5(0.02)$ \\
Dyslipidemia $(\%)$ & $35.7(0.02)$ & $36.7(0.02)$ \\
$\quad$ Type 2 diabetes $(\%)$ & $16.0(0.01)$ & $13.7(0.02)$ \\
Daily medication costs & & \\
Hypertension & $\$ 1.19(0.06)$ & $\$ 1.37(0.1)$ \\
Dyslipidemia & $\$ 1.21(0.08)$ & $\$ 1.38(0.12)$ \\
Type 2 diabetes & $\$ 0.08(0.03)$ & $\$ 0.07(0.03)$ \\
Total & $\$ 2.47(0.11)$ & $\$ 2.83(0.17)$ \\
\hline
\end{tabular}

ITT intention to treat

the Brazier and Roberts algorithm, the imputed QoL scores were 0.797 and 0.803 for the placebo and Qsymia arms, respectively. Largely due to improvements in PCS scores, both groups saw statistically significant improvements in QoL at 56 weeks, 0.009 and 0.027, respectively, resulting in an adjusted improvement of 0.021 for those on Qsymia recommended dose ( $p<0.01$; Table 2$)$.

\subsection{Cost Offsets}

Over the trial period, daily concomitant medication costs per person for antihypertensive, lipid-lowering, and anti- diabetic medications increased by $\$ 0.15$ for placebo and decreased by $\$ 0.22$ for the Qsymia recommended dose arm. This suggests a combined daily saving of $\$ 0.34$ on average for those on the Qsymia recommended dose compared to placebo (Table 3). Annualized, this equates to $\$ 123$ in savings (\$61 for the first year).

\subsection{Costs}

Trial data revealed that the cost of the Qsymia recommended dose is $\$ 1,498$ per participant for 1 year. Physician appointments cost $\$ 89$ each, for a total of \$178 [31]. In the first year, the total payer cost of the Qsymia recommended dose (Qsymia medication costs less cost offsets) was $\$ 1,615$ per participant (Table 4).

\subsection{Base-Case ICER}

Using our base-case assumption of 1 year on the Qsymia recommended dose, with 6 months of benefits in the first year and full benefits linearly decaying to zero by the end of year 3, the ICER is $\$ 48,340$ (all ICERs rounded to the nearest $\$ 10)$.

\subsection{One-Way Sensitivity Analysis}

In a sensitivity analysis where patients remain on the Qsymia recommended dose for 2 years with 6 months of benefits in the first year, full benefits in the second year, and benefits linearly decaying to zero by the end of year four, the ICER increases to $\$ 59,080$ per QALY gained

Table 2 SF-12 Physical Health Summary Scores, Mental Health Summary Scores and quality of life for ITT, mean (SE)

\begin{tabular}{llc}
\hline SF-12 Physical Component Score (PCS) & Placebo $(n=949)$ & Qsymia recommended dose $(n=470)$ \\
\hline Baseline & $44.92(0.27)$ & $45.63(0.38)$ \\
Unadjusted mean change 56 weeks with LOCF & $1.58^{* *}(0.25)$ & $3.59^{* *}(0.33)$ \\
Adjusted change with LOCF 56 weeks ${ }^{\mathrm{a}}$ & & $2.28^{* *}(0.38)$ \\
\hline SF-12 Mental Component Score (MCS) & Placebo $(n=949)$ & Qsymia recommended dose $(n=470)$ \\
\hline Baseline & $53.90(0.26)$ & $54.14(0.35)$ \\
Unadjusted mean change 56 weeks with LOCF & $-0.15(0.24)$ & $-0.13(0.38)$ \\
Adjusted change with LOCF 56 weeks ${ }^{\mathrm{a}}$ & & $0.12(0.38)$ \\
\hline Quality of life & Placebo $(n=954)$ & Qsymia recommended dose $(n=473)$ \\
\hline Baseline & $0.797(0.004)$ & $0.803(0.005)$ \\
Unadjusted mean change 56 weeks with LOCF & $0.009 *(0.003)$ & $0.027^{* *}(0.005)$ \\
Adjusted change 56 weeks ${ }^{\mathrm{a}}$ & Ref. & $0.021^{* *}(0.005)$ \\
\hline LOCF & &
\end{tabular}

LOCF last observation carried forward, ITT intention to treat

$* p<0.05, * * p<0.01$

a ANCOVA with change in outcome as the dependent variable and treatment arm, sex, mean-centered baseline weight, and mean-centered baseline outcome as independent variables 
Table 3 Daily concomitant medication costs for ITT, mean (SE)

\begin{tabular}{lll}
\hline $\begin{array}{l}\text { Daily concomitant medication } \\
\text { costs }\end{array}$ & $\begin{array}{l}\text { Placebo } \\
(n=979)\end{array}$ & $\begin{array}{l}\text { Qsymia } \\
\text { recommended } \\
(n=488)\end{array}$ \\
\hline Baseline (\$) & $2.47(0.12)$ & $2.83(0.17)$ \\
Unadjusted mean change & $\begin{array}{c}0.15^{* *} \\
(0.05)\end{array}$ & $-0.22^{* *}(0.07)$ \\
56 weeks with LOCF $(\$)$ & Ref. & $-0.34^{*}(0.09)$ \\
$\begin{array}{l}\text { Adjusted change with LOCF } \\
56 \text { weeks }(\$)^{\mathrm{a}}\end{array}$ & & \\
\hline
\end{tabular}

$L O C F$ last observation carried forward, ITT intention to treat

$* p<0.05, * * p<0.01$

a ANCOVA with change in outcome as the dependent variable and treatment arm, sex, mean-centered baseline weight, and mean-centered baseline outcome as independent variables

Table 4 Annualized recommended Qsymia costs and cost offsets for ITT

\begin{tabular}{lr}
\hline & \multicolumn{1}{c}{ Costs } \\
\hline Qsymia prescription cost & $\$ 1,498$ \\
Physician appointments & $\$ 178$ \\
Annualized medication cost savings & $-\$ 61$ \\
Total payer perspective & $\$ 1,615$ \\
\hline
\end{tabular}

(Table 5). One-way sensitivity analyses reveal that the assumption of benefit persistence beyond trial cessation has the largest effect on the ICER. Assuming no residual benefit increases the ICER from $\$ 48,340$ in the base case ( 2 years post cessation benefits) to $\$ 152,030$, whereas allowing for 3 years of benefits post cessation decreases the ICER to $\$ 35,270$. The ICER was also increased substantially by taking the lower confidence interval of effectiveness, to $\$ 96,040$, or increasing the prescription costs by $25 \%$ to $\$ 60,420$ per QALY gained. Excluding the medication offset or changing the discount rate did not substantially change the ICER. One year of Qsymia with $100 \%$ benefits and costs, assuming no post cessation benefits, generated an ICER of $\$ 73,120$ (Table 5).

\subsection{Probabilistic Sensitivity Analysis}

For our base case, $54 \%$ of simulations suggest that the cost-effectiveness of the Qsymia recommended dose intervention relative to control is below $\$ 50,000$ per QALY, and $95 \%$ of simulations suggest that it is below $\$ 80,000$.

\subsection{Completers}

Considering the completers sample, the ICER compared to the placebo was $\$ 47,690$ for the base case. Results for the ITT and completer samples were similar because the
Table 5 One-way sensitivity analyses ITT

\begin{tabular}{|c|c|}
\hline & ICER \\
\hline $\begin{array}{l}\text { Base case ( } 1 \text { year on Qsymia, benefits linearly decline to } \\
\text { zero for } 2 \text { years post medication cessation) }\end{array}$ & $\$ 48,340$ \\
\hline \multicolumn{2}{|l|}{ Benefits linearly decline post medication cessation } \\
\hline 0 years (no residual benefits) & $\$ 152,030$ \\
\hline 1 year & $\$ 74,480$ \\
\hline 3 years & $\$ 35,270$ \\
\hline Base case but 2 years on Qsymia & $\$ 59,080$ \\
\hline \multicolumn{2}{|l|}{ Effectiveness assumptions $^{\mathrm{a}}$} \\
\hline Lower CI of QoL & $\$ 96,040$ \\
\hline Upper CI of QoL & $\$ 32,290$ \\
\hline Excluding medication offsets & $\$ 54,120$ \\
\hline \multicolumn{2}{|l|}{ Cost assumptions } \\
\hline Prescription $25 \%$ higher than base case & $\$ 60,420$ \\
\hline Prescription $25 \%$ lower than base case & $\$ 36,250$ \\
\hline \multicolumn{2}{|l|}{ Discount rate (base case $3.5 \%$ ) } \\
\hline $5 \%$ & $\$ 48,980$ \\
\hline $1 \%$ & $\$ 47,260$ \\
\hline Full costs and benefits in year 1 only & $\$ 73,120$ \\
\hline
\end{tabular}

increased cost of the Qsymia recommended dose among completers was similar to the increased QoL benefits (compared to the ITT sample, costs for Qsymia were $17 \%$ higher and QoL benefits were $15 \%$ greater for completers). Detailed results for completers are available in the ESM.

\section{Discussion}

This paper presented the incremental cost-effectiveness (cost per quality-adjusted life year gained) of the recommended dose of Qsymia plus diet and exercise relative to diet and exercise alone (plus placebo). While there is no explicit threshold for what is cost-effective in the US, there are several thresholds frequently cited as indicative of good value for money. The most frequently cited threshold in the US is $\$ 50,000$ per QALY [32]. NICE, the agency that makes coverage recommendations for the National Health Service in the United Kingdom, accepts as cost-effective those interventions with an ICER below $£ 20,000$ (US\$32,000) per QALY, or $£ 30,000$ (US\$48,000) per QALY with strong reasons $(£ 1=$ USD $\$ 1.61$ [33]) [34].

Our base case, which assumes individuals remain on the drug for 1 year and that benefits linearly decay to zero in the 2 years following drug cessation, produced an ICER for the Qsymia recommended dose of $\$ 48,340$, with probabilistic sensitivity analysis revealing that $54 \%$ of our base- 
case simulations were below $\$ 50,000$. Assuming a post benefit decay of only 1 year increased the ICER to $\$ 74,480$ per QALY gained. These results suggest that the Qsymia recommended dose plus diet and exercise may be costeffective, depending on the time on Qsymia medication and whether QoL benefits persist 2 years beyond medication cessation.

This analysis is based solely on the extrapolation of within-trial results and an assumption as to how long QoL benefits would be sustained post treatment. Because the duration that QoL benefits persist beyond the cessation of Qsymia is currently unknown, we modeled sensitivity analyses ranging from no residual benefits to benefits linearly decaying to zero 3 years beyond medication cessation. Future research should explore the average time on Qsymia and the duration that weight loss and QoL benefits persist post Qsymia cessation using real-world data.

This analysis has several limitations. First, because we do not employ a disease progression model, we cannot model potential longer-term savings in QoL or mortality that may result from a lower likelihood of disease progression. Second, because the Qsymia trial did not collect data on healthcare utilization, aside from select medications, our cost offset analysis is limited to reductions in the usage and cost of these medications. Further, there was no significant difference in daily medication costs between the placebo group and Qsymia recommended dose at trial cessation. Given the lower medication cost at baseline for the placebo arm, it is possible that the comparative reduction in medication costs for Qsymia may reflect a regression to the mean. However, it is also possible that incorporating additional longer-term benefits and/or cost offsets would suggest even greater cost-effectiveness results than those reported here. Third, due to the trial design, we were limited to modeling one dosage for the entire trial period, as opposed to following clinician guidelines for dose titration and stoppage rules. The FDA recommends that patients should start on Qsymia $3.75 \mathrm{mg} /$ $23 \mathrm{mg}$ daily for 14 days, then increase to Qsymia $7.5 \mathrm{mg} /$ $46 \mathrm{mg}$ daily (recommended dose) for 12 weeks. If $3 \%$ weight loss is not achieved after 12 weeks on Qsymia $7.5 \mathrm{mg} / 46 \mathrm{mg}$ dose, then it should be discontinued or escalated to Qsymia 11.25/69 for 14 days and then Qsymia $15 / 92$ for 12 weeks. If $5 \%$ weight loss is not achieved after 12 weeks on the Qsymia 15/92 dose, therapy should be discontinued as described in the label [16]. Whether or not this recommendation is followed is unclear, but the 2013 data indicate that roughly $35 \%$ of the volume of Qsymia is from the Qsymia 3.75/23 dose, $56 \%$ from the Qsymia 7.5/ 46 dose, and only $9 \%$ from the Qsymia 11.25/69 and Qsymia 15/92 doses [22]. Due to the trial design, it is not possible to analyze the effects of titration. We chose to conduct the cost-effectiveness analysis focusing on the
Qsymia 7.5/46 daily dosage, herein described as the recommended dose, because in practice few patients titrate to a higher dose after the run-in period. Finally, although our base-case results are based on LOCF, subsequent analyses using baseline carry forward and multiple imputation (MI) had almost no impact on the results; QoL estimates were identical to the fourth decimal in each case.

\section{Conclusion}

Given the health and cost consequences of obesity, payers are looking to identify cost-effective treatments. These results reveal that Qsymia may be cost-effective for overweight and obese individuals with two or more co-morbidities if individuals remain on the drug for extended periods and if QoL benefits are maintained post medication cessation. Quantifying these outcomes using real-world data should be an area of future research.

Acknowledgments Will Liu, Michael Schwiers, and Vincent Wu provided data assistance.

Funding VIVUS, Inc.

Conflict of interest statement This study was conducted under contract between VIVUS, Inc. and Duke-NUS Graduate Medical School, Singapore. EF is a paid consultant of VIVUS, Inc. SK is a paid employee of VIVUS, Inc. EK has no conflict of interest.

Author contributions EF and SK were involved in the concept and design, funding, and supervision of the project. SK obtained the data. $\mathrm{EF}$ and EK were involved in the analysis and interpretation of data, statistical analysis, project administration, and the drafting of the manuscript. SK provided comments and revisions. EF acts as overall guarantor of the manuscript.

Open Access This article is distributed under the terms of the Creative Commons Attribution Noncommercial License which permits any noncommercial use, distribution, and reproduction in any medium, provided the original author(s) and the source are credited.

\section{References}

1. World Health Organization. WHO Consultation. Obesity: preventing and managing the global epidemic. Geneva: World Health Organization; 2000. p 894.

2. WHO. Obesity and overweight. 2013. http://www.who.int/ mediacentre/factsheets/fs311/en/. Accessed 11 Oct 2013.

3. Finkelstein EA, Trogdon JG, Cohen JW, Dietz W. Annual medical spending attributable to obesity: payer-and service-specific estimates. Health Aff. 2009;28(5):w822-31.

4. Finkelstein EA, daCosta DiBonaventura M, Burgess SM, Hale BC. The costs of obesity in the workplace. J Occup Environ Med. 2010;52(10):971-6.

5. NHLBI Obesity Education Initiative Panel. Clinical guidelines on the identification, evaluation, and treatment of overweight and obesity in adults. Bethesda: NHLBI; 2004. 
6. Franz MJ, VanWormer JJ, Crain AL, Boucher JL, Histon T, Caplan W, et al. Weight-loss outcomes: a systematic review and meta-analysis of weight-loss clinical trials with a minimum 1-year follow-up. J Am Diet Assoc. 2007;107(10):1755-67.

7. Turk MW, Yang K, Hravnak M, Sereika SM, Ewing LJ, Burke LE. Randomized clinical trials of weight-loss maintenance: a review. J Cardiovasc Nurs. 2009;24(1):58.

8. Picot J, Jones J, Colquitt JL, Gospodarevskaya E, Loveman E, Baxter $L$ et al. The clinical effectiveness and cost-effectiveness of bariatric (weight loss) surgery for obesity: a systematic review and economic evaluation. Health Technol Assess (Winchester, England). 2009;13(41):1-190, 215-357, iii-iv. doi:10.3310/ hta13410.

9. Livingston EH. The incidence of bariatric surgery has plateaued in the US. Am J Surg. 2010;200(3):378-85.

10. Centers for Disease Control and Prevention. Adult obesity facts. 2014. http://www.cdc.gov/obesity/data/adult.html. Accessed 23 April 2014.

11. U.S. Census Bureau, Population Division. Annual estimates of the resident population by single year of age and sex for the United States: April 1, 2010 to July 1, 2012. 2013. http:// factfinder2.census.gov/faces/tableservices/jsf/pages/productview. xhtml?src=bkmk. Accessed 23 April 2014.

12. Centers for Disease Control and Prevention. Cardiac valvulopathy associated with exposure to fenfluramine or dexfenfluramine: U.S. Department of Health and Human Services interim public health recommendations, November 1997. Morb Mortal Wkly Rep. 1997;46(45):1061-6.

13. US FDA. Meridia (sibutramine): market withdrawal due to risk of serious cardiovascular events. 2010. http://www.fda.gov/safety/ medwatch/safetyinformation/safetyalertsforhumanmedicalprodu cts/ucm228830.htm. Accessed 9 Sept 2013.

14. Padwal R, Li S, Lau D. Long-term pharmacotherapy for overweight and obesity: a systematic review and meta-analysis of randomized controlled trials. Int J Obes. 2003;27(12):1437-46.

15. Padwal RS, Majumdar SR. Drug treatments for obesity: orlistat, sibutramine, and rimonabant. Lancet. 2007;369(9555):71.

16. US FDA. Medication guide: Qsymia. 2012. http://www.fda.gov/ downloads/Drugs/DrugSafety/UCM312590.pdf. Accessed 23 April 2014.

17. Gadde KM, Allison DB, Ryan DH, Peterson CA, Troupin B, Schwiers ML, et al. Effects of low-dose, controlled-release, phentermine plus topiramate combination on weight and associated comorbidities in overweight and obese adults (CONQUER): a randomised, placebo-controlled, phase 3 trial. Lancet. 2011;377(9774):1341-52.

18. Garvey WT, Ryan DH, Look M, Gadde KM, Allison DB, Peterson CA, et al. Two-year sustained weight loss and metabolic benefits with controlled-release phentermine/topiramate in obese and overweight adults (SEQUEL): a randomized, placebo-controlled, phase 3 extension study. Am J Clin Nutr. 2012;95(2): 297-308.
19. Fox J. Medicare should, but cannot, consider cost: legal impediments to a sound policy. Buffalo Law Rev. 2005;53.

20. Kolber MS. Opacity and cost effectiveness analysis in Medicare coverage decisions: health policy encounters administrative law. Food Drug Law J. 2009;64:515.

21. UnitedHealth Group. UnitedHealth Group launches innovative alliance providing free access to programs that help prevent and control diabetes and obesity. 2010. http://www.unitedhealth group.com/Newsroom/Articles/News/UnitedHealth\%20Group/ 2010/0414DiabetesObesity.aspx. Accessed 8 May 2014.

22. Health IMS. National prescription audit. Ambler: IMS America; 2012.

23. Ware JE, Kosinski M, Dewey JE, Gandek B. SF-36 health survey: manual and interpretation guide. Lincoln: Quality Metric Inc.; 2000.

24. Brazier JE, Roberts J. The estimation of a preference-based measure of health from the SF-12. Med Care. 2004;42(9):851-9.

25. Hay JW, Katon WJ, Ell K, Lee P-J, Guterman JJ. Cost-effectiveness analysis of collaborative care management of major depression among low-income, predominantly hispanics with diabetes. Value Health. 2012;15(2):249-54.

26. Reynolds MR, Zimetbaum P, Josephson ME, Ellis E, Danilov T, Cohen DJ. Cost-effectiveness of radiofrequency catheter ablation compared with antiarrhythmic drug therapy for paroxysmal atrial fibrillation. Circ Arrhythm Electrophysiol. 2009;2(4):362-9.

27. Katon W, Russo J, Sherbourne C, Stein MB, Craske M, Fan M-Y, et al. Incremental cost-effectiveness of a collaborative care intervention for panic disorder. Psychol Med. 2006;36(03): 353-63.

28. Bensink M, Wootton R, Irving $\mathrm{H}$, Hallahan A, Theodoros D, Russell $\mathrm{T}$, et al. Investigating the cost-effectiveness of videotelephone based support for newly diagnosed paediatric oncology patients and their families: design of a randomised controlled trial. BMC Health Serv Res. 2007;7(1):38.

29. StataCorp. Stata Statistical Software, release 13. College Station: StataCorp LP; 2013.

30. Medi-Span. Price RX database. 2013. http://www.medispan.com/ drug-pricing-analysis-pricerx.aspx. Accessed 27 Sept 2013.

31. Medicare. Fee schedule, payment and reimbursement benefit guideline, CPT code billing. Office visit CPT, E\&M code fee schedule-Florida Medicare. 2013. http://www.medicare paymentandreimbursement.com/2013/01/office-visit-cpt-e-codefee-schedule.html. Accessed 17 Jan 2014.

32. Grosse SD. Assessing cost-effectiveness in healthcare: history of the $\$ 50,000$ per QALY threshold. Expert Rev Pharmacoecon Outcomes Res. 2008;8(2):165-78. doi:10.1586/14737167.8.2. 165 .

33. XE.com. XE live exchange rates. 2014. http://www.xe.com. Accessed 24 Feb 2014.

34. NICE. Chapter 8: Linking clinical guidelines to other NICE guidance. In: The guidelines manual. London: NICE; 2012. 